\title{
When Primary Users Whisper: A Preliminary Analysis on Correlations of Population-Traffic Dynamics
}

\author{
Alexandros Palaios, Maria Michalopoulou, Janne Riihijärvi, Petri Mähönen \\ Institute for Networked Systems, RWTH Aachen University, Aachen, Germany \\ email: apa@inets.rwth-aachen.de
}

\begin{abstract}
In this paper we report the preliminary results from the large-scale spectrum measurement campaign based study to find out correlations between spectrum use and socio-economical data. We use two large spectrum usage datasets that have been collected from metropolitan London and Paris, and correlate the spectrum usage of GSM, UMTS, and $2.4 \mathrm{GHz}$ ISM band against the local population density and location type in those cities. We show that although the spectrum usage, measured in duty cycle and average received power in bands, in general shows the correlation with the population density, one has to also consider the type of the city area as an extra parameter. One of the key surprising findings is that that there are also areas, where the spectrum usage is uncorrelated, or even negatively correlated, with the increasing population density. This shows that spectrum usage models need to be more detailed than just population density conditioned. Moreover, the measurement campaign and found correlations show that one can derive useful information on the network structure and conduct useful test driving campaigns with a surprisingly small amount of data points.
\end{abstract}

\section{INTRODUCTION}

The trend towards enabling a cooperative or secondary use of spectrum has raised the necessity to acquire stronger understanding of the current spectrum usage. Exploiting the spectrum usage patterns in the most widely used frequency bands can provide useful knowledge for the design and deployment strategies of secondary networks, developing attractive and sustainable cooperation schemes, and for spectrum modeling.

The characterization of spectrum occupancy is a complex and difficult task due mainly to its highly dynamic nature. Therefore, the existence of a potential interaction between spectrum usage and population will be particularly interesting and useful, especially because of the fact that population densities are generally well known and reasonably static with respect to time. Also capability to predict better the needed capacity and deployment strategy would be important not only for industry, but could enable also academia to build better synthetic models for research purposes. Although the hypothesis that the population density should correspond with the spectrum usage level is quite common, there is only very limited amount of publicly available research that is based on experimental data.

In the present paper we analyze the correlations between the data gathered by two large spectrum measurement campaigns and the underlying population densities. More specifically, this work reports the initial analysis of the partial spatial data, acquired from our measurement campaigns, from metropolitan London and Paris. In both cities we have selected measurement locations so that they cover different population densities and land-use types (urban business core area, residential, and suburban). Our analysis goal is twofold: first, to study the correlations between received power measurements and population densities in diverse environments as a preliminary step in understanding the structure of wireless services, spectrum utilization, and socio-economical data. Second, we want to understand if socioeconomical data, or population density alone, could be used as an indicator for required number of spectrum measurement points and type of network deployments that are needed to satisfy demand. The spectrum usage has been studied during the last decade by different academic and research groups. Most of the campaigns have been geographically limited with severely constrained spatiotemporal resolution. One of the seminal multisite measurement was done my McHenry et al. [1]. Other notable measurement campaigns include, e.g. [2]-[11]. In the cellular bands an early primary user study was conducted by Willkom et al. [6], and the alter work by Paul et al. [12] is also an important contribution. One of the rare similar recent works, but based on anonymized cellular call data is by Becker et al. [13]. One of the key contributions of our measurement campaign is that we are looking the typical situation at the city areas having highly calibrated measurement platforms measuring data roughly at the human head height, and having high spatial-resolution with large number of radio samples in each location. Thus the underlying radio data is high quality, and this is then combined with the socioeconomical data; in the case of this paper we limit ourselves to consider only population density and rough land area use.

This study builds upon our earlier work on understanding the spatial dynamics of radio propagation in different environments, consisting of studies where we considered different neighborhoods in the same city [10] and cities across different countries [11], [14]. For this type of work, the consistency of the measurement equipment is critical, thus special attention was paid during all the measurements in order to allow comparison of the captured measurements later on. Furthermore, in [15] we have considered the correlations between population 


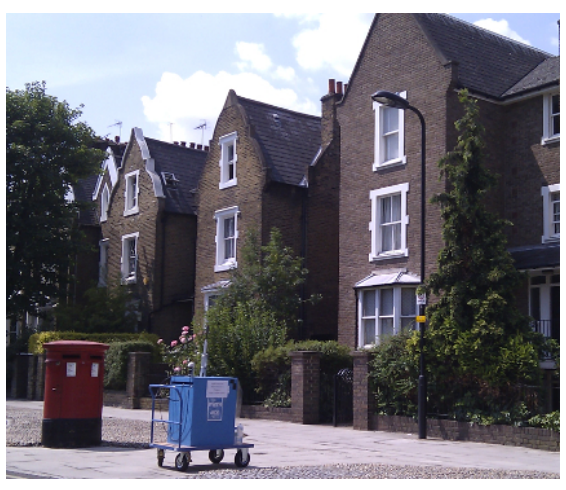

(a) Residential Area, London

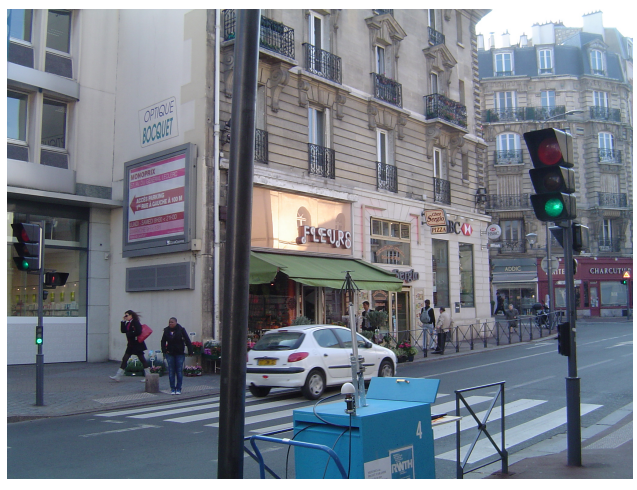

(b) Business Area, Paris

Fig. 1. Two of the measurement locations in London and Paris.

densities and the cellular network usage in Germany at a countrywide level. Although a completely different data set is used here, it is of a great interest to observe that some conclusions drawn in [15] are reinforced here.

The remainder of the paper is organized as follows. In Section II we describe in detail the measurement campaign as far as the equipment, setup and measurement sites are concerned. Section III presents the mathematical tools and metrics involved in the present work. In Section IV we illustrate and discuss the results. Finally, the paper is concluded in Section $\mathrm{V}$.

\section{Measurement Configuration SETTINGS AND LOCATIONS}

In this work we focus on studying the dynamics of spectrum usage in different neighborhoods by deploying two portable spectrum analyzers, built in a big blue casing, as these are depicted in Figure 1.

The spectrum analyzers were located at street level with the receiver antenna height at $1.75 \mathrm{~m}$, approximately at the same height at which a mobile device is held during usage. For a more detailed description of the measurement campaign the interested reader is referred to [10]. In this work, we present results collected by two mobile measurement platforms that captured a large number of samples during the day in the GSM 900, GSM 1800, UMTS, and WLAN bands.

For each measurement point metadata was also recorded, containing precise time and localization information, as well as images of the neighborhood. The localization information allows us later on to associate measurement files with the corresponding population count and socioeconomical data of the area under study.

The used measurement platforms are identical and carefully calibrated in order to guarantee that the measurement results from different locations can be compared. The measurement configuration is a trade-off process; there is no single configuration that suffices to capture all different aspects of the radio environment at the highest precision. The selection of the configuration parameters influences the characteristics of the radio environment that shall be captured, for example, whether frequency resolution shall be favored over time resolution. Here we discuss briefly about the important parameters and the selections we made:

- Frequency spans: We selected to cover the four particular frequency bands, namely GSM 900, GSM 1800, UMTS, and $2.4 \mathrm{GHz}$ ISM-band that most notably includes WLAN (Wi-Fi) traffic as these are the four most used bands regarding today's wireless services. This selection allowed us to achieve a time resolution of a few seconds. If we targeted for a larger frequency span, the achieved time resolution would be significantly lower.

- Resolution Bandwith: $100 \mathrm{kHz}$, as this is very close to typical channels in use nowadays. We find this to be a fair compromise between accumulated noise and required resolution for this comparative analysis.

- Detector: RMS (Root-Mean-Square) as it is the most appropriate type for energy measurements.

- Cables losses were measured. We were consistently using identical cables of fixed size and length, so that they could be swapped during the measurement campaign if needed - without altering the sensitivity of the system.

- Sensitivity: The spectrum analyzers we used calculate the frequency dependent gains automatically reporting directly the compensated RMS value. The overall achieved sensitivity was calculated to be $-95.0 \mathrm{dBm} / 100 \mathrm{kHz}$. In post-processing we slightly increased the threshold to $-99.0 \mathrm{dBm} / 100 \mathrm{kHz}$ in order to account for any additional small discrepancies.

- Spectrum Analyzer: Rohde \& Schwarz FSL6

- Antenna: AOR DA3200, supporting to the total range of $25 \mathrm{MHz}-3000 \mathrm{MHz}$

A list and description of the measurement locations is illustrated in Table I.

The population data has been obtained from the Global Population Dataset LandScan (2006) ${ }^{\mathrm{TM}}$ [16]. The dataset comprises a collection of GIS raster maps that is, in essence, population data given on a latitude/longitude grid. The dataset has a spatial resolution of 30 arc seconds, which corresponds to a 
TABLE I

MEASUREMENT LOCATIONS, DESCRIPTIONS AND DETAILS.

\begin{tabular}{llllcc}
\hline Date & Location & City & Description & $\begin{array}{c}\text { Measurement } \\
\text { locations }\end{array}$ & $\begin{array}{c}\text { Measurement } \\
\text { duration [h] }\end{array}$ \\
\hline Wed, Oct 13, 2010 & $\begin{array}{l}\text { Issy-les } \\
\text { Moulineaux }\end{array}$ & Paris & $\begin{array}{l}\text { At the heart of the Val de } \\
\text { Seine business district }\end{array}$ & 68 & 5.5 \\
\hline Sat, July 02, 2011 & $\begin{array}{l}\text { Oxford } \\
\text { Street }\end{array}$ & London & London's main Shopping Area & 35 & 6 \\
Sat, July 02, 2011 & $\begin{array}{l}\text { Trafalgar } \\
\text { Square }\end{array}$ & London & Touristic and Night-life Area & 22 & 3 \\
Mon, July 04, 2011 & $\begin{array}{l}\text { Residential } \\
\text { Area }\end{array}$ & London & $\begin{array}{l}\text { De Beauvoir, District of Lon- } \\
\text { don in } \\
\text { Woodford, North East London }\end{array}$ & 28 & 26 \\
Suburban & London & $\begin{array}{l}\text { Wrea } \\
\text { suburb }\end{array}$ & & 26 \\
\hline
\end{tabular}

cell size of approximately $1 \mathrm{~km} \times 1 \mathrm{~km}$ near the equator and finer elsewhere. The given values in the data set are population counts per grid cell. For obtaining a one to one correspondence between the measurement locations and the population counts, we match each measurement location to the nearest cell center of the population grid. Although a higher resolution population density data could have been useful for us, our sensitivity analysis indicates that the current resolution is enough for our modeling purposes.

Due to the fact that our measurement locations have a higher spatial density compared to the population data, some of the measurement points correspond to the same entry of the population database. This mapping means that we might report sometimes lower correlations than the real underlying population vs. spectrum usage, however the error is not a serious and we, in fact, prefer to be conservative in our correlation estimation. In this first approach of analysis we did not conduct any filtering on our measurement data, but leave that for our future work.

\section{Mathematical Tools and Metrics}

\section{A. Correlation Metrics}

We evaluate the correlation between two random variables $X$ and $Y$ by means of the Pearson's correlation coefficient

$$
r_{X Y}=\frac{E[(X-E(X))(Y-E(Y))]}{\sigma_{X} \sigma_{Y}},
$$

where $E$ is the expectation value operator, and $\sigma_{X}, \sigma_{Y}$ are the standard deviations of $X$ and $Y$, respectively.

The Spearman's correlation coefficient is a measure of the rank correlation of two random variables $X$ and $Y$ and is equivalent to the Pearson's correlation coefficient between the ranked variables. Therefore, similarly to Pearson's correlation, the Spearman's rank correlation ranges also from +1 to -1 . If the random variable values $X_{i}$ and $Y_{i}$ are converted to ranks $x_{i}$ and $y_{i}$, then the Spearman's correlation coefficient is defined as

$$
\rho_{X Y}=\frac{\sum_{i}\left(x_{i}-E[x]\right)\left(y_{i}-E[y]\right)}{\sqrt{\sum_{i}\left(x_{i}-E[x]\right)^{2}\left(y_{i}-E[y]\right)^{2}}} .
$$

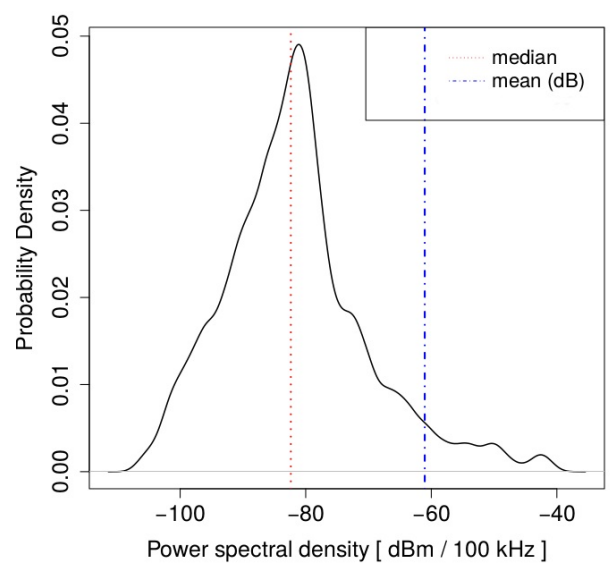

Fig. 2. The different traffic metrics on captured sample data in Oxford Street. The Duty Cycle is $0.89 \%$

The Spearman's correlation is a measure of monotone association between two random variables, that is, if $Y$ tends to increase with $X$ the Spearman's correlation is positive and if $Y$ tends to decrease when $X$ increases the Spearman's correlation is negative. A perfect Spearman's correlation of +1 (or -1) indicates an increasing (or decreasing) monotonic relationship between $X$ and $Y$.

\section{B. Metric Selection}

For the energy measurements we captured, we had to decide which metrics are more appropriate for the purposes of the present analysis, namely for correlating them with the population information. We considered three potential quantities: the mean value of the measured power at a location in decibel scale, the median of the measured power in decibel scale, and the duty cycle. The three metrics are depicted in Figure 2 for an arbitrary measurement location.

In order to obtain the mean value in $\mathrm{dBm}$ we convert the measured power values from $\mathrm{dBm}$ to Watts, we calculate the mean value and then convert the resulting value back to $\mathrm{dBm}$. 
TABLE II

Cellular Bands

\begin{tabular}{llcc}
\hline Location & Band & $r_{X Y}$ & $\rho_{X Y}$ \\
\hline Oxford Street & GSM DL 900 & -0.10 & -0.16 \\
Oxford Street & GSM UL 900 & -0.38 & -0.48 \\
Oxford Street & GSM DL 1800 & -0.19 & -0.38 \\
Trafalgar Square & GSM UL 900 & -0.44 & -0.39 \\
Issy-les Moulineaux & GSM DL 1800 & -0.16 & -0.13 \\
Issy-les Moulineaux & UMTS DL & -0.41 & -0.38 \\
Oxford Street & UMTS DL & -0.07 & -0.32 \\
\hline
\end{tabular}

Although the mean value is the most widely used metric when results from more than one experiments or measurements need to be combined to a single value, in our case its usage would not be a sensible choice due to the outlier values (i.e., very high received powers) that add a long tail to the probability density function of the measured power. This effect is mainly a result of the multipath component, which is dominant in urban environments. As illustrated in Figure 2, as a result of the long tail, the mean value in $\mathrm{dB}$ is not a suitable quantity to represent the plotted density function.

The median, however, is known to work well on samples with skewed tails and, unlike the mean, its robustness does not suffer from the existense of outliers. We have also a benefit of having a very large number of measurement points.

The same holds for the duty cycle, which can be defined as follows

$$
\mathrm{DC}=\sum \frac{\mathrm{On}}{\mathrm{On}+\mathrm{Off}}, \text { On }>-95 \mathrm{dBm} / 100 \mathrm{kHz},
$$

and basically renders any measurement as zero or one based on a selected threshold (used threshold was $-95 \mathrm{dBm} / 100 \mathrm{kHz}$ ). Therefore, it treats all the outliers in the same way as all measurements that fall above the threshold.

\section{REsults}

\section{A. Urban High Terminal Density Environments}

We start our analysis by focusing on data that was collected from metropolitan core areas, meaning with this those city center areas that have a very high population density during the business hours. We call these areas high terminal density domains. It is important to note this distinction, the underlying population density in the population databases is not as high as the real transient (terminal) population density. This is due to the fact as that areas of the city attract large number of business and touristy usage that is not properly coded into population data which is normally based on registered living (home) address data.

In the Oxford Street (Table II), the GSM 900 downlink (DL) shows weak negative correlations, while the uplink exhibits even stronger negative correlations. The capture of the uplink channel depends on the exact location of the measurement equipment and as our setup was at the street level, the activity of the people passing by the box was captured. Similar correlations were also estimated at a different urban location, namely the Trafalgar square, with the Pearson's and Spearman's coefficients being -0.44 and -0.39 respectively.

In the GSM 1800 band, in Oxford Street, slightly larger correlations were found. It is interesting to observe that the Spearman's coefficient is larger in both bands, GSM 900 and GSM 1800, as it is not practically affected by outliers, which are typical in high terminal density environments, where multipath components become dominant. We also report results from the Issy-les Moulineaux district in Paris, where the GSM 1800 downlink band shows similarly weak correlations as well.

The UMTS bands show similar negative correlations between the population density data and the duty cycle. Specifically, in Paris the Pearson's and Spearman's correlations are -0.41 and -0.38 respectively. Also, in the Oxford street, the Spearman's coefficient was found negative, with a value of -0.32 .

We should note that as the measurement setup was immobile, during the measurement period the multipath dynamics were also captured [17]. The multipath components are time and location specific since they are caused by physical objects in the environment, such as cars moving on the street. We will discuss more extensively the effects of the multipath component in Section IV. For the moment, we would like to mark that it is indeed interesting that even in this case some correlation trends remain visible. Of course, additional work needs to be conducted toward this direction in the future in order to investigate more precisely the extent to which the multipath components affect correlations.

As discussed in the beginning of the section, the real peak hour terminal density is likely to be much higher than underlying (living) population density. This will cause bias in the calculated correlation, which is likely to induce negative component to the correlation. However, there can be also other reasons that contribute to the negative correlations. One of them is that the core urban areas lack capacity. One should note that in order to satisfy the high capacity requirements, providers tend to decrease the dimensions of the cells. More cells in an area means that each cell can potentially interfere with more cells. Consequently, each cell needs to be more power-confined, and lower transmission levels are allowed. In our results this trend is visible to some extent since the measured power seems to exhibit a negative correlation with the population counts.

These results for urban areas are also in agreement with the findings reported in [15], where the correlations between population and cellular traffic in Germany were investigated. It was also reported that within city areas the cellular traffic load and the population densities were not correlated, although correlations existed at a country-wide scale.

\section{B. Residential Environments}

In this subsection we look at residential/suburban areas, and more specifically a residential district within London and a suburban area at the city outskirts. For the suburban area we found that there is a strong positive correlation between 
population and activity of primary users. Results are shown in Table III. For example, for the GSM 900 downlink we estimated coefficients of 0.83 and 0.87 for the Pearson's and Spearman's correlation, respectively. This a clear indicator of a strong positive correlation between the population and the duty cycle.

TABLE III

RESIDENTIAL AREAS

\begin{tabular}{llcc}
\hline Location & Band & $r_{X Y}$ & $\rho_{X Y}$ \\
\hline Residential & GSM DL 900 & 0.23 & 0.16 \\
Suburban & GSM DL 900 & 0.83 & 0.87 \\
Residential & GSM DL 1800 & 0.26 & 0.20 \\
Suburban & GSM DL 1800 & 0.77 & 0.73 \\
Residential & UMTS DL & 0.23 & 0.06 \\
Suburban & UMTS DL & -0.21 & -0.16 \\
\hline
\end{tabular}

Similar results we observe for the GSM 1800 band (with statistically high coefficients of 0.77 and 0.73 ). The UMTS band though gave us small negative correlations. This can be explained by the demands of UMTS technologies being small in such areas at the time the measurements were conducted. Moreover, the GSM 900 is being refarmed for UMTS activity (see [10]), so the typical UMTS band does not have enough activity for correlations to be found.

The strong positive correlations in the suburban environment reveals information about the structure of the cellular network deployments, with the most straightforward speculation being that only a few base stations provide coverage over the whole area. This is of course expected as due to the relatively low capacity demands in such areas, the primary problem for the service provider is the coverage, and not the capacity. This means that - in comparison to urban areas - fewer cells can cover the same region by just increasing the transmitted powers in the cells.

In the residential area in London (Table III) for the GSM 900 downlink (DL) we found weak positive correlations of 0.23 and 0.16 for Pearson's and Spearman's coefficients, respectively. Similar results we observed also for the GSM 1800 downlink. The UMTS downlink band appears to have very weak positive correlations. Compared to the other two environments we discussed already, that is, the high terminal density core areas and suburban ones, the residential area seems to be somewhere in the middle of those two different propagation environments (very dynamic against an almost static one).

\section{On the Propagation Dynamics in Metropolitan Core}

In this section we want to note that the lack or small correlation coefficients does not necessarily mean that correlations do not exist. One should be careful and understand the limitations of the measurement campaign. For example, the limitations imposed by the propagation environment might be such a factor, especially in the 'crowdy' environments. In Figure 3 we show the captured energy levels and their corresponding Cumulative Distribution Functions (CDF) for two measurement points in the Oxford street. We have selected two measurement points that are only 150 meters apart.

Even in such short distances, the captured data of the two points, reveal the vast differences as the dominant land usage changes. Specifically, there are differences on the order of $-50 \mathrm{dBm}$ between the points. This can be partially explained from the cell sizes that are small but still such fluctuations within the same measurement point, on the order of $-40 \mathrm{dBm}$ reveal how harsh fluctuations the multipath components can cause.

These fluctuations are caused by local reflectors, including cars, traffic, people, and large windows, that influence the calculation of the correlations by increasing the noise in the captured measurement set.

In our earlier work, see [18], we have shown that is possible to remove the effects of the multipath components during the measurement period but this is a very time consuming process. This can be also partially done in the post processing but we leave this for future work.

For the suburban area, we have also selected two measurement point that are about $150 \mathrm{~m}$ apart. In Figure 3 we show the difference compared to the two locations on the Oxford street. The two measurement points are almost identical, where only small changes are found. As the data set has fewer of outliers, in the form of multipath makes the correlations calculations

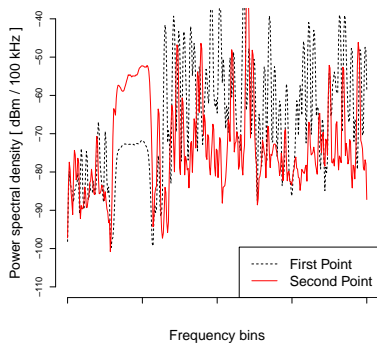

(a) Oxford Street, London, Power Spectral Density

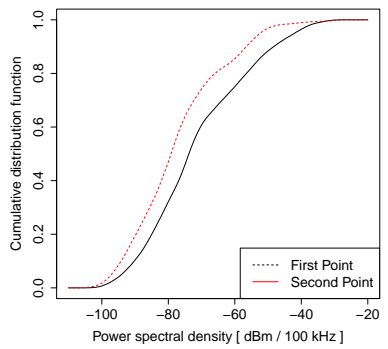

(b) Oxford Street, London,CDF plot (c) Suburban Area, London, Power Spectral Density

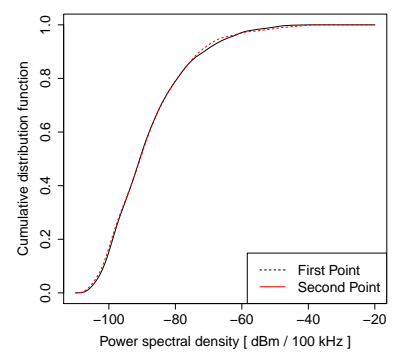

(d) Suburban Area, London, CDF plot

Fig. 3. The power spectral density of two locations in Oxford Street and two locations in Suburban Area that are not more than $150 \mathrm{~m}$ apart. 
TABLE IV

THE ESTIMATED CORRELATION COEFFICIENTS FOR A SMALL NUMBER OF SAMPLES

\begin{tabular}{llcccc}
\hline Location & Band & $r_{X Y}$ & Estimated $r_{X Y}$ & $\rho_{X Y}$ & Estimated $\rho_{X Y}$ \\
\hline Issy-les Moulineaux & UMTS DL & -0.41 & -0.42 & -0.38 & -0.32 \\
Suburban & GSM DL 900 & 0.83 & 0.80 & 0.87 & 0.83 \\
Oxford & GSM DL 1800 & -0.19 & -0.20 & -0.38 & -0.40 \\
\hline
\end{tabular}

an easier task (this is also expected as the environment around the receiver is almost static during measurement).

\section{Lightweight Correlations Calculations}

As it was already discussed, the existence of correlations, has a potential to reveal information about the underlying network deployments. Even though more research is needed on this topic, it is quite probable that this type of information can be beneficial to secondary users and generally to the research community on developing realistic models. The cell size is a factor that put limits on the interference, as smaller transmission levels are needed because many more cells are now susceptible to the emitted power of secondary users.

One should also note that capability to calculate correlations in near real-time, is something that would also drastically reduce time and complexity needed by test drives. In this paper we have effectively demonstrated a simple and lightweight method for calculating correlation estimates based on energy measurements. This is fast as we do not need large numbers of samples, which relaxes requirements on the energy consumption of the RF component, the memory size and the processing power thus casting also possibility to use handheld devices for this type of measurements.

As we have discussed in Section III, the median is not significantly affected by outliers. We combine that simple metric with a very small number of samples. So in our small example one can just use just the median of the captured values and estimate quite accurately the correlations just after 10 measurements that are collected within 3 minutes time-period.

In Table IV, we present the estimated values on a very small number of samples. Interesting is that with a few samples the correlation coefficients acquired are very close to the ones we presented above. That renders of course simpler approaches more tractable for such type of applications.

\section{Conclusions}

We have shown that there is a strong correlation with the population density and spectrum usage in suburban regions. This finding also corresponds well with studies done by using call statistics from cellular networks, see Becker et al. [13] and references therein. However, we report that the actual correlation levels can be different between different regions especially if one considers urban core areas against residential/suburban areas. As discussed, this scaling difference is likely to be partially caused by a natural bias in the population density databases, by data having different usage patterns than voice call services, and by the increased data usage that tends to drive networks to their capacity limits. We are able to confirm the claim of Becker et al. that mobile data can be used to predict where people live, and we are doing this by using just spectrum monitoring data instead of using actual call data.

One of the indications is that the spectrum monitoring data is correlated with socio-economical data, but one has to be careful in the analysis. Single parameter universal laws do not seem to be easy to generate, but actually one needs to consider multi-parameter information in order to generate predictive models. We are currently working on extending our analysis. This includes analyzing more data points, applying spatial-statistical methods in the data analysis, and filtering multipath components out of our spectrum data. Moreover, we plan to correlate our datasets against other sociological datasets. We consider also to study correlations between spectrum measurements and call information data in the future campaigns. The finding of correlation structures should be useful for research community on testing their models and making generic deployment scenarios. We are currently also studying the possibility to provide later open access to our raw data sets.

\section{ACKNOWLEDGMENT}

We would like to that DFG (Deutsche Forschungsgemeinschaft) for financial support through UMIC Resarch Center at the RWTH Aachen University. We also acknowledge a partial financial support from European Union through ACROPOLIS Network of Excellence and ARAGORN projects. The authors want to thank colleagues from KCL, London in their help on the measurements, especially Dr. Oliver Holland, and help of OrangeLabs staff in logistics during our Paris measurement campaign. One of us (PM) acknowledges also DFG support through RWTH Fellowship.

\section{REFERENCES}

[1] M. A. McHenry and D. McCloskey, "Multi-band, multi-location spectrum occupancy measurements," in Proc. of International Symposium on Advanced Radio Technologies (ISART), Boulder, CO, USA, March 2006.

[2] M. A. McHenry, P. A. Tenhula, D. McCloskey, D. A. Roberson, and C. S. Hood, "Chicago spectrum occupancy measurements \& analysis and a long-term studies proposal," in Proceedings of the first international workshop on Technology and policy for accessing spectrum. New York, NY, USA: ACM, 2006. [Online]. Available: http://doi.acm.org/10.1145/1234388.1234389

[3] M. Islam, C. Koh, S. W. Oh, X. Qing, Y. Lai, C. Wang, Y.-C. Liang, B. Toh, F. Chin, G. Tan, and W. Toh, "Spectrum Survey in Singapore: Occupancy Measurements and Analyses," in Proc. of CROWNCOM'08, 2008, pp. 1-7.

[4] V. Valenta, R. Marsalek, G. Baudoin, M. Villegas, M. Suarez, and F. Robert, "Survey on spectrum utilization in Europe: Measurements, analyses and observations," in Proc. of CROWNCOM'10, 2010, pp. 1- 
[5] R. Bacchus, T. Taher, K. Zdunek, and D. Roberson, "Spectrum Utilization Study in Support of Dynamic Spectrum Access for Public Safety," in Proc. of IEEE DySPAN 2010, 2010, pp. 1-11.

[6] D. Willkomm, S. Machiraju, J. Bolot, and A. Wolisz, "Primary user behavior in cellular networks and implications for dynamic spectrum access," IEEE Communications Magazine, vol. 47, no. 3, pp. 88-95, 2009.

[7] A. Palaios, J. Riihijärvi, O. Holland, and Mähönen, "A week in London: Spectrum usage in Metropolitan London," in Proc. of IEEE PIMRC 2013, September 2013.

[8] S. Yin, D. Chen, Q. Zhang, M. Liu, and S. Li, "Mining Spectrum Usage Data: A Large-Scale Spectrum Measurement Study," IEEE Transactions on Mobile Computing, vol. 11, no. 6, pp. 1033-1046, 2012.

[9] M. Wellens, J. Riihijärvi, and P. Mähönen, "Spatial statistics and models of spectrum use," Computer Communications, vol. 32, no. 18, pp. 19982011, 2009.

[10] A. Palaios, J. Riihijärvi, O. Holland, A. Achtzehn, and P. Mähönen, "Measurements of Spectrum Use in London: Exploratory Data Analysis and Study of Temporal, Spatial and Frequency-Domain Dynamics," in Proc. of IEEE DySPAN 2012, October 2012.

[11] A. Palaios, J. Riihijärvi, P. Mähönen, V. Atanasovski, L. Gavrilovska, P. Van Wesemael, A. Dejonghe, and P. Scheele, "Two days of spectrum use in Europe," in Proc. of CROWNCOM'12, June 2012.

[12] U. Paul, A. Subramanian, M. Buddhikot, and S. Das, "Understanding traffic dynamics in cellular data networks," in Proc. of IEEE INFOCOM 2011, 2011, pp. 882-890.

[13] R. Becker, R. Caceres, K. Hanson, J. Loh, S. Urbanek, A. Varshavsky, and C. Volinsky, "A Tale of One City: Using Cellular Network Data for Urban Planning," Pervasive Computing, IEEE, vol. 10, no. 4, pp. 18-26, 2011.

[14] A. Palaios, J. Riihijärvi, P. Mähönen et al., "Two days of European Spectrum: Preliminary analysis of concurrent spectrum use in seven European sites in GSM and ISM bands," in Proc. of IEEE ICC 2013, June 2013.

[15] M. Michalopoulou, J. Riihijarvi, and P. Mahonen, "Towards characterizing primary usage in cellular networks: A traffic-based study," in Proc. of IEEE DySPAN 2011, 2011, pp. 652-655.

[16] "LandScan (2006) ${ }^{\mathrm{TM}}$ High Resolution global Population Data Set copyrighted by UT-Battelle, LLC, operator of Oak Ridge National Laboratory." http://www.ornl.gov/sci/landscan/, [Online; last visited on January 21, 2014.].

[17] T. S. Rappaport, Wireless Communications: Principles and Practice $2^{\text {nd }}$ Edition. Prentice Hall, 2002.

[18] N. Perpinias, A. Palaios, J. Riihijärvi, and P. Mähönen, "Impact of the path loss model on the spatial structure of shadow fading: A measurement-based study in a sub-urban environment," in Proc. of IEEE ICC 2014, 2014. 